\title{
A Survey of Adaptive Streaming over HTTP
}

\author{
Shukla Ravi ${ }^{1}$, Prof. Darshana Shah ${ }^{2}$ \\ ${ }^{1}$ (EC Department, Gujarat Technological University, India) \\ 2(EC Department, Gujarat Technological University, India)
}

\begin{abstract}
Adaptive streaming over Hyper-Text Transport protocol (HTTP) is the new trend in video delivery on the Internet and is expected to be supported by consumer electronic devices such as Blu-ray players and DVRs. Proprietary solutions have been around for a couple of years and standardization efforts are entering the final stage. Adaptive bit rate streaming is a technique used in streaming multimedia over computer networks. While in the past most video streaming technologies utilized streaming protocols such $R T P$ with RTSP, today's adaptive streaming technologies are almost exclusively based on HTTP[1]and designed to work efficiently over large distributed HTTP networks such as the Internet. Experiments confirm that our algorithm outperforms popular segmentation techniques and saves $10 \%$ of bandwidth on average for the same objective quality levels. This saving is significant given the volume of traffic that video delivery generates every day on the Internet. I
\end{abstract}

Keyword :HTTP, DASH, streaming

\section{Introduction:}

In recent years an obvious need emerged to improve the way the video content is delivered over the Internet. As multimedia consumption became significant part of the overall traffic usage, coupled with the emergence of mobile browsing content, providers needed a reliable and robust way to deliver content to end users. In order to simplify the process of content management and delivery and overcome limitations of streaming video through firewalls, leading companies adopted streaming over HTTP as predominant way of video streaming. Since video service providers have no control over bandwidth

a vailability over the Internet, adaptive streaming is employed to provide uninterrupted video delivery even as the available bandwidth varies. These solutions have been around for a couple of years with most popular implementations being Smooth Streaming, HTTP Dynamic Streaming [1], and HTTP Live Streaming HLS [2]. Segments are offered at different bit rate levels. It works by detecting a user's bandwidth and CPU capacity in real time and adjusting the quality of a video stream accordingly. It requires the use of an encoder which can encode a single source video at multiple bit rates. The player client switches between streaming the different encodings depending on available resources. The result: very little buffering, fast start time and a good experience for both high-end and low-end connections.

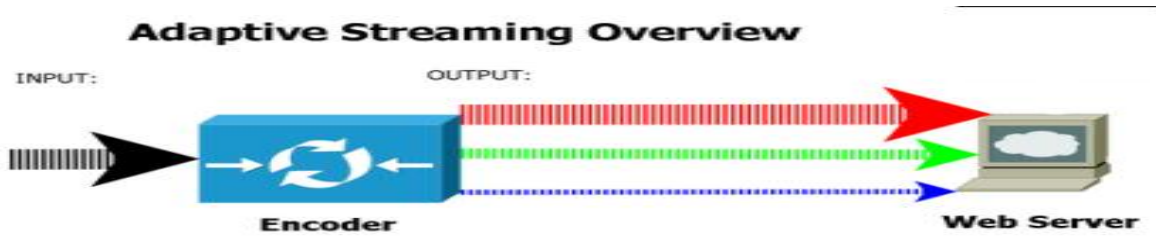

Fig.1

\section{Streaming Process:-}

Streaming" multimedia allows the user to begin viewing video clips stored on our server, without first downloading the entire file. After a brief period of initializing and buffering, the file will begin to stream or play in real time. Your picture quality will be determined by your available bandwidth (download speed) and computer processor speed. Users with older processors and slower modem connections will have more dropped frames and pixelization. Streaming media is multimedia that is constantly received by and presented to an enduser while being delivered by a provider. Its verb form, "to stream", refers to the process of delivering media in this manner; the term refers to the delivery method of the medium rather than the medium itself. A client media player can begin playing the data (such as a movie) before the entire file has been transmitted. Distinguishing delivery method from the media distributed applies specifically to telecommunications networks, as most other delivery systems are either inherently streaming (e.g., radio, television) or inherently non streaming (e.g., books, video cassettes, audio CDs). For example, in the 1930s, elevator music was among the earliest popularly available streaming media; nowadays Internet television is a common form of streamed media. The term 
"streaming media" can live closed captioning, stock ticker, and real-time text, which are all considered "streaming text". The term "streaming" was first used in the early 1990s as a better description for video on demand on IP networks; at the time such video was usually referred to as "store and forward video",[1] which was misleading nomenclature. Live streaming, which refers to content delivered live over the Internet, requires a camera for the media, an encoder to digitize the content, a media publisher, and a content delivery network to distribute and deliver the content.

The primary technical issues related to streaming were:

* having enough CPU power and bus bandwidth to support the required data rates

* Creating low-latency interrupt paths in the operating system to prevent buffer under run.

However, computer networks were still limited, and media were usually delivered over non-streaming channels, such as by downloading a digital file from a remote server and then saving it to a local drive on the end user's computer or storing it as a digital file and playing it back from CD-ROMs.

\section{Streaming Bandwidth And Storage:-}

A broadband speed of $2.5 \mathrm{M}$ bit/s or more is recommended for streaming movies, for example to an Ro ku , Apple TV, Google TV or a Sony TV Blu-ray Disc Player, 10 M bit/s for High Definition content.

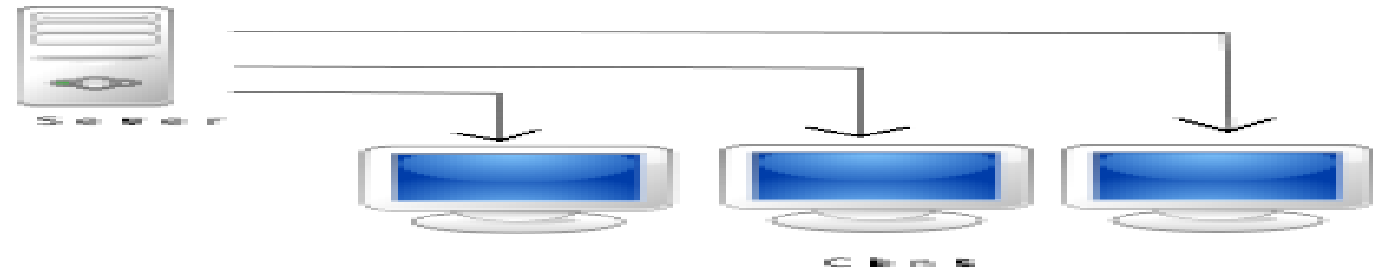

Fig.2 server and client

Unicast connections require multiple connections from the same streaming server even when it streams the same content

Streaming media storage size is calculated from the streaming bandwidth and length of the media using the following formula (for a single user and file):

storage size (in megabytes) $=$ length (in seconds) bit rate (in bit/s) / $(8 * 1024 * 1024)$

Real world example:

One hour of video encoded at $300 \mathrm{k}$ bit/s (this is a typical broadband video as of 2005 and it is usually encoded in a $320 * 240$ pixels window size) will be:

$(3,600 \mathrm{~s} * 300,000 \mathrm{bit} / \mathrm{s}) /(8 * 1024 * 1024)$ requires around $128 \mathrm{MB}$ of storage.

If the file is stored on a server for on-demand streaming and this stream is viewed by 1,000 people at the same time using a Uni cast protocol, the requirement is:

$300 \mathrm{k} \mathrm{bit} / \mathrm{s} * 1,000=300,000 \mathrm{k} \mathrm{bit} / \mathrm{s}=300 \mathrm{M} \mathrm{bit} / \mathrm{s}$ of bandwidth

This is equivalent to around $135 \mathrm{~GB}$ per hour. Using a multicast protocol the server sends out only a single stream that is common to all users. Therefore such a stream would only use $300 \mathrm{kbit} / \mathrm{s}$ of serving bandwidth. See below for more information on these protocols. The calculation for live streaming is similar.

Assumptions: speed at the encoder, is $500 \mathrm{kbit} / \mathrm{s}$.

If the show lasts for 3 hours with 3,000 viewers, then the calculation is:

Number of MBs transferred = encoder speed (in bit/s) *number of seconds *number of viewers / $(8 * 1024 * 1024)$ Number of MBs transferred $=500 \times 1024$ (bit/s) $\times * 3 * 3,600$ ( = 3 hours) *3,000 (no of viewers) / $(8 * 1024 * 1024)=1,977,539 \mathrm{MB}$

\section{Adaptive Streaming:}

Adaptive bit rate streaming is a technique used in streaming multimedia over computer networks. While in the past most video streaming technologies utilized streaming protocols such RTP with RTSP, today's adaptive streaming technologies are almost exclusively based on HTTP[1] and designed to work efficiently over large distributed HTTP networks such as the Internet.

It works by detecting a user's bandwidth and CPU capacity in real time and adjusting the quality of a video stream accordingly. It requires the use of an encoder which can encode a single source video at multiple bit rates. The player client[2] switches between streaming the different encodings depending on available resources.[3] "The result: very little buffering, fast start time and a good experience for both high-end and lowend connections. 
More specifically, and as the implementations in use today are, adaptive bit rate streaming is method of video streaming over HTTP where the source content is encoded at multiple bit rates, then each of the different bit rate streams are segmented into small multi-second parts The streaming client is made aware of the available streams at differing bit rates, and segments of the streams by a manifest file. When starting, the client requests the segments from the lowest bit rate stream. If the client finds the download speed is greater than the bit rate of the segment downloaded, then it will request the next higher bit rate segments. Later, if the client finds the download speed for a segment is lower than the bit rate for the segment, and therefore the network throughput has deteriorated, then it will request a lower bit rate segment. The segment size can vary depending on the particular implementation, but they are typically between two (2) and ten (10) seconds.

\section{DASH(Dynamic Adaptive Streaming over HTTP):}

Dynamic Adaptive Streaming over HTTP (DASH), also known as MPEG-DASH, enables high quality streaming of media content over the Internet delivered from conventional HTTP web servers. Similar to Apple's HTTP Live Streaming (HLS) solution, MPEG-DASH works by breaking the content into a sequence of small HTTP-based file segments, each segment containing a short interval of playback time of a content that is potentially many hours in duration, such as a movie or the live broadcast of a sports event. The content is made available at a variety of different bit rates, i.e., alternative segments encoded at different bit rates covering aligned short intervals of play back time are made available. As the content is played back by an MPEG-DASH client, the client automatically selects from the alternatives the next segment to download and play back based on current network conditions. The client selects the segment with the highest bit rate possible that can be downloaded in time for play back without causing stalls or re buffering events in the playback. Thus, an MPEGDASH client can seamlessly adapt to changing network.

DASH is an adaptive bit rate streaming technology where a multimedia file is partitioned into one or more segments and delivered to a client using HTTP. A media presentation description (MPD) describes segment information (timing, URL, media characteristics such as video resolution and bit rates). Segments can contain any media data, however the specification provides specific guidance and formats for use with two types of containers: MPEG-4 file format or MPEG-2 Transport Stream.

DASH is audio/video codec agnostic. One or more representations (i.e., versions at different resolutions or bit rates) of multimedia files are typically available, and selection can be made based on network conditions, device capabilities and user preferences, enabling adaptive bit rate streaming and Qo E fairness. DASH is also agnostic to the underlying application layer protocol. Thus, DASH can be used with any protocol, conditions, and provide high quality play back without stalls or re buffering events.

MPEG-DASH is the first adaptive bit-rate HTTP-based streaming solution that is an international standard[1] MPEG-DASH leverages the already widely deployed HTTP web server infrastructure that is used for delivery of essentially all Internet content. It allows devices such as Internet connected televisions, TV set-top boxes, desktop computers, smart phones, tablets, etc. to consume multimedia content (video, TV, radio...) delivered via the Internet, coping with variable Internet receiving conditions, thanks to its adaptive streaming technology.

\section{Benefits Of Adaptive Bit Rate Streaming:}

Consumers of streaming media experience the highest quality material when adaptive bit rate streaming is used because the user's network and playback conditions are automatically adapted to at any given time under changing conditions.

The media and entertainment industry are the main beneficiaries of adaptive bit rate streaming. As the video space grows exponentially, content delivery networks and video providers can provide customers with a superior viewing experience. Adaptive bit rate technology requires additional encoding, but by putting the client in charge, it simplifies the overall workflow and creates better results.

\section{Conclusion}

The presentation novel approach for coding and segmentation of video content for transmission by adaptive streams over HTTP. Optimized Algorithms fills essential for the preparation of the contents of such streaming Adaptation is again gaining popularity on the Internet. Along with other techniques for delivery and adaptation user side this approach may introduce non substantial savings

\section{References}

[1]. S. Akhshabi, A.C. Begen, and C.Dovrolis, "An experimental evaluation of rateadaptation algorithms in adaptive streaming over HTTP," Proc. of the second annual ACM conference on Multimedia systems, pp.157-168, Feb. 2011.

[2]. Velibor Adzic, Student Member, IEEE, Hari Kalva, Senior Member, IEEE and Borko Furht" Optimizing Video Encoding for Adaptive Streaming over HTTP"

[3]. Chang-Su Kim1, Bong-Im Jang2 and Hoe-Kyung Jung3" "A Study on Dynamic Adaptive Streaming System over HTTP for Various Streaming Services." 
[4]. Heiko Schwarz, Detlev Marpe, Member, IEEE, and Thomas Wiegand, Member, IEEE“ Overview of the Scalable Video Coding Extension of the H.264/AVC Standard"

[5]. I. Sodagar,ÿ?The MPEG-DASH Standard for Multimedia Streaming Over the Internet?, IEEE Multimedia, Oct-Nov 2011.

[6]. T. Stockhammer, I. Sodagar, ?MPEG DASH: The Enabler Standard for Video Deliver Over The Open Internet,? IBC Conference 2011, Sept 2011.

[7]. ̈I. Sodagar and H. Pyle, ?Reinventing multimedia delivery with MPEG-DASH?,̈̈SPIE Applications of Digital Image Processing XXXIV, Sept 2011.

[8]. T. Stockhammer: ?Dynamic Adaptive Streaming over HTTP-Design Priciples and Standards? In: MMSys ?11: Proceedings of the second annual ACM conference on Multimedia systems New York, NY, USA: ACM Press, Feb 2011 , S. 133-144.

[9]. http://www.rfidradio.com/?p=13

[10]. www.ideaonboard.urg/uvc 